PROCEEDINGS OF THE

AMERICAN MATHEMATICAL SOCIETY

Volume 136, Number 1, January 2008, Pages 103-110

S 0002-9939(07)08860-0

Article electronically published on September 24, 2007

\title{
THE GLOBAL ATTRACTIVITY OF THE RATIONAL DIFFERENCE EQUATION $y_{n}=A+\left(\frac{y_{n-k}}{y_{n-m}}\right)^{p}$
}

\author{
KENNETH S. BERENHAUT, JOHN D. FOLEY, AND STEVO STEVIĆ \\ (Communicated by Carmen C. Chicone)
}

AbstraCt. This paper studies the behavior of positive solutions of the recursive equation

$$
y_{n}=A+\left(\frac{y_{n-k}}{y_{n-m}}\right)^{p}, \quad n=0,1,2, \ldots,
$$

with $y_{-s}, y_{-s+1}, \ldots, y_{-1} \in(0, \infty)$ and $k, m \in\{1,2,3,4, \ldots\}$, where $s=$ $\max \{k, m\}$. We prove that if $\operatorname{gcd}(k, m)=1$, and $p \leq \min \{1,(A+1) / 2\}$, then $y_{n}$ tends to $A+1$. This complements several results in the recent literature, including the main result in K. S. Berenhaut, J. D. Foley and S. Stević, The global attractivity of the rational difference equation $y_{n}=1+\frac{y_{n-k}}{y_{n-m}}$, Proc. Amer. Math. Soc., 135 (2007) 1133-1140.

\section{INTRODUCTION}

This paper studies the behavior of positive solutions of the recursive equation

$$
y_{n}=A+\left(\frac{y_{n-k}}{y_{n-m}}\right)^{p}, n=0,1, \ldots,
$$

with $y_{-s}, y_{-s+1}, \ldots, y_{-1} \in(0, \infty)$ and $k, m \in\{1,2,3,4, \ldots\}$, where $s=\max \{k, m\}$.

The case $k=m$ is trivial, so from now on we will assume that $k \neq m$.

Note that if $g=\operatorname{gcd}(k, m)>1$, then $\left\{y_{i}\right\}$ can be separated into $g$ different equations of the form

$$
y_{n}^{(j)}=A+\left(\frac{y_{n-\frac{k}{g}}^{(j)}}{y_{n-\frac{m}{g}}^{(j)}}\right)^{p},
$$

where $j \in\{1,2, \ldots, g\}$. Hence, we may assume that $\operatorname{gcd}(k, m)=1$.

The study of properties of rational and nonlinear difference equations has been an area of intense interest in recent years; cf. [1]-25] and the references therein.

There is a relatively long history in studying equation (11). For example, for $p=1$, the case $k=2, m=1$ was studied in 2 by Amleh et al., the case $k \in \mathbb{N}$, $m=1$ was studied by DeVault et al. in [11, and the case $A>1, k=1, m \in \mathbb{N}$ was studied by Stević in 20]. The investigation of global stability and periodicity of

Received by the editors April 18, 2006 and in revised form, July 31, 2006.

2000 Mathematics Subject Classification. Primary 39A10, 39A11.

Key words and phrases. Rational difference equation, stability.

The first author acknowledges financial support from a Sterge Faculty Fellowship. 
positive solutions of equation (10), for the case $p=A=1, k, m \in \mathbb{N}$ was completed by results in [3] and [15]; see also [17] and [21].

The study of the case $p>1$ was suggested in [14, where the authors noted that some results from [2] for the case $p=1, k=2, m=1$, can be translated to the case $p>1, k=2, m=1$. The first results for the case $p<1$ were given in 23. The existence of monotone solutions, for the case $p>0$ and $A>-1$ was shown in [5] by developing the technique from [6, 7, 8, 9, 10, 24] and [25]. Equations in papers [4] and [12] were investigated by transforming them into some special cases of equation (11).

The linearized equation associated with equation (1) for the case $k=2$ and $m=1$ is

$$
(A+1) z_{n}+p z_{n-1}-p z_{n-2}=0,
$$

and its characteristic roots are

$$
\lambda_{1}=\frac{-p+\sqrt{p^{2}+4 p(A+1)}}{2(A+1)} \quad \text { and } \quad \lambda_{2}=\frac{-p-\sqrt{p^{2}+4 p(A+1)}}{2(A+1)} .
$$

By some simple calculation we obtain

$$
\left|\lambda_{1}\right|=\frac{2 p}{p+\sqrt{p^{2}+4 p(A+1)}}<1
$$

for every $p, A>0$.

On the other hand, we have that

$$
\left|\lambda_{2}\right|<1 \Longleftrightarrow 2 p<A+1 \text {. }
$$

Hence, when $2 p<A+1$ equation (10) for the case $k=2$ and $m=1$ is locally asymptotically stable by the Linearized Stability Theorem.

Motivated by this local stability result, in 22. Stević has posed the following conjecture.

Conjecture 1. If $k=2, m=1$ and $p, A \in(0,1)$ are such that $p<(A+1) / 2$, then every positive solution of equation (11) converges to the unique equilibrium $A+1$.

Among other results, here we confirm the conjecture by proving that the following holds true for every $k, m \in \mathbb{N}, 0<A<1$ and $0<p \leq(A+1) / 2$.

Theorem 1. Suppose that $m, k \geq 1$, and that $p, A$ are positive numbers satisfying $0<A<1$ and $0<p \leq(A+1) / 2$. If the sequence $\left\{y_{i}\right\}$ satisfies (11) with $y_{-s}, y_{-s+1}, \ldots, y_{-1} \in(0, \infty)$ where $s=\max \{m, k\}$, then, $\left\{y_{i}\right\}$ converges to the unique equilibrium $A+1$.

Remark 1 (The case $k$ even and $m$ odd). Note that the general characteristic equation associated with the linearized equation for equation (1) is

$$
(A+1) \lambda^{s}+p \lambda^{s-m}-p \lambda^{s-k}=0,
$$

and for $k$ even and $m$ odd, equation (3) has a real root $\lambda_{0}<-1$, when $p>(A+1) / 2$. To see this, suppose that $p>(A+1) / 2$, and set

$$
f(\lambda)=A+1+\frac{p}{\lambda^{m}}-\frac{p}{\lambda^{k}} .
$$

Now, note that $f(-1)=A+1-2 p<0$, and for $\lambda<-1$,

$$
f(\lambda)=A+1-\frac{p}{|\lambda|^{m}}-\frac{p}{|\lambda|^{k}}>A+1-\frac{2 p}{|\lambda|^{\min \{m, k\}}}>0,
$$


for sufficiently large $|\lambda|$.

Hence, by the continuity of the function $f$ on the interval $(-\infty,-1)$ it follows that $f(\lambda)=0$ for some $\lambda \in(-\infty,-1)$, as required.

Thus, by the Linearized Stability Theorem, the positive equilibrium $\bar{y}=A+1$ of equation (1) is not stable, in this case. This fact in conjunction with Theorem 1 gives a full characterization of stability for the case $k$ even and $m$ odd, for $A, p \in$ $(0,1)$.

The paper proceeds as follows. In Section 2, we introduce some preliminary lemmas and notation. Section 3 is devoted to global stability, where among other results we give a proof of Theorem 1 .

\section{Preliminaries And notation}

In this section, we introduce some preliminary lemmas and notation.

First, consider the simple transformed sequence $\left\{z_{i}\right\}$ defined by $z_{n}=y_{n}-A$, for $n \geq-s$. Then, equation (11) becomes

$$
z_{n}=\left(\frac{A+z_{n-k}}{A+z_{n-m}}\right)^{p}
$$

for $n \geq 0$.

Now, define $\left\{z_{i}^{*}\right\}$ by

$$
z_{i}^{*}= \begin{cases}z_{i}, & \text { if } z_{i} \geq 1 \\ \frac{1}{z_{i}}, & \text { otherwise. }\end{cases}
$$

The following elementary lemma will be useful.

Lemma 1. If $x>1$ and $0<A<1$, then

$$
\left(\frac{A+x}{A+1 / x}\right)^{\frac{A+1}{2}} \leq x
$$

with the inequality if and only if $x=1$, and if $x \geq 1$ and $A>1$, then the reverse inequality to inequality (8) holds.

Proof. Assume first that $A \in(0,1)$. Then the inequality in (8) is equivalent to

$$
g_{A}(x) \stackrel{\text { def }}{=}(A+1) \ln \left(\frac{A+x}{A x+1}\right)-(1-A) \ln x \leq 0 .
$$

Note that

$$
\lim _{x \rightarrow+\infty} g_{A}(x)=-\infty \text { and } g_{A}(1)=0 .
$$

By some simple calculations we obtain that

$$
g_{A}^{\prime}(x)=-\frac{A(x-1)^{2}(1-A)}{(A+x)(A x+1) x}<0,
$$

when $x \neq 1$, since $A \in(0,1)$.

Hence, $g_{A}(x)$ is decreasing, and thus by (10) is negative on the interval $(1, \infty)$.

Now, assume that $A>1$. Then $\lim _{x \rightarrow+\infty} g_{A}(x)=+\infty$ and $g_{A}(1)=0$. On the other hand, by (11) it follows that $g_{A}^{\prime}(x)>0$, from which the desired inequality follows.

Remark 2. Note that if $A=1$ the inequality in (8) becomes equality. 
Next we prove a contraction lemma which will be helpful in showing convergence of solutions in the transformed space obtained through (77).

Lemma 2. Suppose $\left\{z_{i}\right\}$ satisfies (6) with $p \leq(A+1) / 2$ and $A \in(0,1]$. Then,

$$
1 \leq z_{n}^{*} \leq \max \left\{z_{n-k}^{*}, z_{n-m}^{*}\right\},
$$

for all $n \geq s$.

Proof. Suppose that $z_{n-k}>z_{n-m}$ and set $x=\max \left\{z_{n-k}^{*}, z_{n-m}^{*}\right\}$. Note that if $z_{n-k} \geq 1$, then $1 \leq z_{n-k} \leq x$ and consequently

$$
1 / x \leq z_{n-k} \leq x
$$

and if $z_{n-k}<1$, then $1 / z_{n-k}=z_{n-k}^{*} \leq x$ from which (13) also holds. Similarly, we have that

$$
1 / x \leq z_{n-m} \leq x .
$$

Then, from (6), (13) and (14), for $n \geq s$, we have that

$$
z_{n}^{*}=z_{n}=\left(\frac{A+z_{n-k}}{A+z_{n-m}}\right)^{p} \leq\left(\frac{A+z_{n-k}}{A+z_{n-m}}\right)^{\frac{A+1}{2}} \leq\left(\frac{A+x}{A+\frac{1}{x}}\right)^{\frac{A+1}{2}} \leq x,
$$

where the final inequality in (15) follows from Lemma 1. Similarly, if $z_{n-k} \leq z_{n-m}$, then

(16) $z_{n}^{*}=\frac{1}{z_{n}}=\left(\frac{A+z_{n-m}}{A+z_{n-k}}\right)^{p} \leq\left(\frac{A+z_{n-m}}{A+z_{n-k}}\right)^{\frac{A+1}{2}} \leq\left(\frac{A+x}{A+\frac{1}{x}}\right)^{\frac{A+1}{2}} \leq x$,

and the lemma is proved.

Now, set

$$
D_{n}=\max _{n-s \leq i \leq n-1}\left\{z_{i}^{*}\right\},
$$

for $n \geq s$.

The following result is a simple consequence of Lemma 2 and (17).

Lemma 3. The sequence $\left\{D_{i}\right\}$ is monotonically nonincreasing in $i$, for $i \geq s$.

Since $D_{i} \geq 1$ for $i \geq s$, Lemma 3 implies that, as $i$ tends to infinity, the sequence $\left\{D_{i}\right\}$ converges to some limit, say $D$, where $D \geq 1$.

Next, we have the following lemma concerning boundedness of solutions to equation (1).

Lemma 4. If $p \in(0,1)$, then every positive solution of equation (11) is bounded.

Proof. First, note that each $n \in \mathbb{N}_{0}$ can be written in the form $l k+i$, for some $l \in \mathbb{N}_{0}$ and $i \in\{0,1, \ldots, k-1\}$. Let $l_{0}=l_{0}(i)$ be the smallest element of $\mathbb{N}_{0}$ such that $l_{0} k+i \geq m$. From (11) and since $y_{n}>A$ for every $n \geq 0$, we have that

$$
y_{l k+i}=A+\frac{y_{(l-1) k+i}^{p}}{y_{l k+i-m}^{p}}<A+\frac{y_{(l-1) k+i}^{p}}{A^{p}},
$$

for every $l \in \mathbb{N}_{0}$ and $i \in\{0,1, \ldots, k-1\}$ such that $l k+i \geq m$. Let $\left(u_{l}^{(i)}\right)_{l \in \mathbb{N}}$ be the solution of the difference equation

$$
u_{l}^{(i)}=A+\frac{u_{l-1}^{(i)}}{A^{p}}, \quad u_{l_{0}}^{(i)}=y_{i\left(l_{0}-1\right)+i} .
$$


By (18) and induction we see that $y_{(l-1) k+i} \leq u_{l}^{(i)}, l \geq l_{0}$. Hence it is enough to prove that the sequences $\left(u_{l}^{(i)}\right)_{l \geq l_{0}}, i \in\{0,1, \ldots, k-1\}$, are bounded.

Since the function

$$
f(x)=A+\frac{x^{p}}{A^{p}}, \quad x \in(0, \infty),
$$

is increasing and concave for $p \in(0,1)$ it follows that there is a unique fixed point $\bar{x}$ of the equation $f(x)=x$ and that the function $f$ satisfies the condition

$$
(f(x)-x)(x-\bar{x})<0, \quad x \in(0, \infty) .
$$

Using this fact it is easy to see that if $u_{l}^{(i)} \in(0, \bar{x}]$ the sequence is nondecreasing and bounded above by $\bar{x}$ and if $u_{l}^{(i)} \geq \bar{x}$, it is nonincreasing and bounded below by

$\bar{x}$. Hence for every $u_{l_{0}}^{(i)} \in(0, \infty)$, each of the sequences $u_{l}^{(i)}, i \in\{0,1, \ldots, k-1\}$, is bounded, from which the result follows.

\section{Convergence of solutions to equation (1)}

In this section, we study the global attractivity of the positive solutions of equation (1). First, we give a proof of Theorem 1

Proof of Theorem 1. Note that it suffices to show that the transformed sequence $\left\{z_{i}^{*}\right\}$ converges to 1 .

By the definition in (17), the values of $D_{i}$ are taken on by entries in the sequence $\left\{z_{j}^{*}\right\}$, and as well, by Lemma $2, z_{i}^{*} \in\left[1, D_{i}\right]$ for $i \geq s$. Now, for any $\epsilon>0$, we can find an $N$ such that

$$
z_{N}^{*} \in[D, D+\epsilon]
$$

and for $i \geq N-s$,

$$
z_{i}^{*} \in[1, D+\epsilon] \text {. }
$$

Note that, similar to (13) and (14), (20) implies that

$$
\frac{1}{D+\epsilon} \leq z_{N-m}, z_{N-k} \leq D+\epsilon \text {. }
$$

We will show that $D=1$, and from this, (7), (17) and the definition of $D$, the result follows.

Now, suppose $D>1$, and note that (19) implies that $z_{N} \neq 1$.

First, consider the case $z_{N}>1$. Then, from (19), we have that

$$
z_{N}=z_{N}^{*} \in[D, D+\epsilon] \text {. }
$$

Solving for $z_{n-k}$ in (6), and employing (22) and (21), gives

$$
\begin{aligned}
D+\epsilon & \geq z_{N-k}=z_{N}^{1 / p}\left(A+z_{N-m}\right)-A \\
& \geq D^{1 / p}\left(A+\frac{1}{D+\epsilon}\right)-A \\
& \geq D^{\frac{2}{A+1}}\left(A+\frac{1}{D+\epsilon}\right)-A .
\end{aligned}
$$

This implies that

$$
\left(\frac{A+D+\epsilon}{A+\frac{1}{D+\epsilon}}\right) \geq D^{\frac{2}{A+1}}
$$


Assume now that $z_{N}<1$. Then,

$$
\frac{1}{z_{N}}=z_{N}^{*} \in[D, D+\epsilon] \text {. }
$$

From (6), and employing (21) and (25), it follows that

$$
\begin{aligned}
D+\epsilon & \geq z_{N-m}=\left(z_{N}^{*}\right)^{1 / p}\left(A+z_{N-k}\right)-A \\
& \geq D^{1 / p}\left(A+\frac{1}{D+\epsilon}\right)-A \\
& \geq D^{\frac{2}{A+1}}\left(A+\frac{1}{D+\epsilon}\right)-A .
\end{aligned}
$$

From (26) we have that (24) holds in this case, as well. Since $\epsilon>0$ was arbitrary and $D>1$, by Lemma 1 we arrive at a contradiction, which implies that $D=1$, and the theorem follows.

Remark 3. Note that the above argument breaks down when $p>(A+1) / 2$. In particular, we have that for $p>(A+1) / 2$,

$$
\left(\frac{A+x}{A+1 / x}\right)^{p}>x
$$

for $x=1+\epsilon$, for sufficiently small $\epsilon>0$. To see this, similar to (9), set

$$
h_{A, p}(x)=p(\ln (A+x)-\ln (A x+1))-(1-p) \ln x,
$$

and note that the condition $h_{A, p}(x)>0$ is equivalent to (27). Now, $h_{A, p}(1)=0$ and

$$
h_{A, p}^{\prime}(1)=\frac{2 p-(A+1)}{(A+1)}>0 .
$$

Hence for sufficiently small $\epsilon>0, h_{A, p}(1+\epsilon)>0$.

The next theorem is devoted to the case $p \in(0,1)$ and $A \geq 1$. It is simpler than Theorem 1 and is essentially a consequence of the boundedness result in Lemma 4 ,

Theorem 2. Suppose that $m, k \geq 1, p \in(0,1)$ and $A \geq 1$. If the sequence $\left\{y_{i}\right\}$ satisfies (11) with $y_{-s}, y_{-s+1}, \ldots, y_{-1} \in(0, \infty)$ where $s=\max \{m, k\}$, then, $\left\{y_{i}\right\}$ converges to the unique equilibrium $A+1$.

Proof. By Lemma 4, every solution $\left\{y_{n}\right\}$ of equation (11) is bounded which implies that there are finite $\lim \inf y_{n}=\lambda$ and $\lim \sup y_{n}=\Lambda$. Assume to the contrary that $\lambda<\Lambda$. Taking the liminf and limsup in (1) it follows that

$$
A+\frac{\lambda^{p}}{\Lambda^{p}} \leq \lambda<\Lambda \leq A+\frac{\Lambda^{p}}{\lambda^{p}} .
$$

From this and since $p \in(0,1)$, it follows that

$$
A \Lambda^{p}+\lambda^{p} \leq \Lambda^{p} \lambda<\Lambda \lambda^{p} \leq A \lambda^{p}+\Lambda^{p}
$$

i.e.,

$$
(A-1) \Lambda^{p}<(A-1) \lambda^{p} .
$$

Since $A \geq 1$, this is impossible. Therefore we have that $\lambda=\Lambda$, which implies the result. 


\section{REFERENCES}

[1] R. M. Abu-Saris and R. DeVault, Global stability of $y_{n+1}=A+\frac{y_{n}}{y_{n-k}}$, Appl. Math. Lett. 16 (2) (2003), 173-178. MR:1962312 (2004c:39037)

[2] A. M. Amleh, E. A. Grove, G. Ladas and D. A. Georgiou, On the recursive sequence $y_{n+1}=\alpha+\frac{y_{n-1}}{y_{n}}$, J. Math. Anal. Appl. 233 (1999), 790-798. MR1689579(2000f:39002)

[3] K. S. Berenhaut, J. D. Foley and S. Stević, The global attractivity of the rational difference equation $y_{n}=1+\frac{y_{n-k}}{y_{n-m}}$, Proc. Amer. Math. Soc., 135 (2007), 1133-1140. MR2262916 (2007f:39006)

[4] K. S. Berenhaut and S. Stević, A note on the difference equation $x_{n+1}=\frac{1}{x_{n} x_{n-1}}+$ $\frac{1}{x_{n-3} x_{n-4}}$, J. Differ. Equations Appl. 11 (14) (2005), 1225-1228. MR2182249

[5] K. S. Berenhaut and S. Stević, On Positive Nonoscillatory Solutions of the Difference Equation $x_{n+1}=\alpha+\frac{x_{n-k}{ }^{p}}{x_{n}{ }^{p}}$, J. Differ. Equations Appl., in press (2005).

[6] L. Berg, Asymptotische Darstellungen und Entwicklungen, Dt. Verlag Wiss., Berlin, 1968. MR0241873 (39:3210)

[7] L. Berg, On the asymptotics of nonlinear difference equations, Z. Anal. Anwendungen 21, No.4, (2002), 1061-1074. MR1957315 (2004b:39015)

[8] L. Berg, Inclusion theorems for non-linear difference equations with applications, J. Differ. Equations Appl. 10 (4) (2004), 399-408. MR2047219 (2004m:39007)

[9] L. Berg, Corrections to "Inclusion theorems for non-linear difference equations with applications," from [3], J. Differ. Equations Appl. 11 (2) (2005), 181-182. MR2114324|(2005h:39007)

[10] L. BERG AND L. V. WOLFERSDORF, On a class of generalized autoconvolution equations of the third kind, Z. Anal. Anwendungen 24, No. 2 (2005), 217-250. MR:2174021 (2006j:45020)

[11] R. DeVault, C. Kent and W. Kosmala, On the recursive sequence $x_{n+1}=p+\frac{\iota_{n-k}}{x_{n}}, J$. Differ. Equations Appl. 9 (8) (2003), 721-730. MR1992905(2004f:39014)

[12] R. DeVault, G. Ladas and S.W. Schultz, On the recursive sequence $x_{n+1}=\frac{A}{x_{n} x_{n-1}}+$ $\frac{1}{x_{n-3} x_{n-4}}$, J. Differ. Equation Appl. 6 (4) (2000), 481-483. MR1785161

[13] H. El-Metwally, E. A. Grove, G. Ladas, and H. D. Voulov, On the global attractivity and the periodic character of some difference equations, J. Diff. Eqn. Appl. 7 (2001), 837-850. MR.1870725 (2003e:39006)

[14] H. M. El-Owaidy, A. M. Ahmed and M. S. Mousa, On asymptotic behaviour of the difference equation $x_{n+1}=\alpha+\frac{x_{n-1}^{p}}{x_{n}^{p}}$, J. Appl. Math. \& Computing 12 (1-2) (2003), 31-37. MR.1976801 (2004a:39011)

[15] E. A. Grove, AND G. Ladas, Periodicities in Nonlinear Difference Equations, Chapman \& Hall/CRC Press, Boca Raton, (2004). MR2193366 (2006j:39002)

[16] V. Kocić AND G. LAdAS. Global behavior of nonlinear difference equations of higher order with applications, Mathematics and its Applications, 256. Kluwer Academic Publishers Group, Dordrecht, 1993. MR.1247956 (94k:39005)

[17] W. T. Patula and H. D. Voulov. On the oscillation and periodic character of a third order rational difference equation. Proc. Amer. Math. Soc. 131 (2003), no. 3, 905-909. MR1937429 (2003j:39008)

[18] S. Stević, Asymptotic behaviour of a sequence defined by iteration with applications, Colloq. Math. 93 (2) (2002), 267-276. MR 1930804 (2003k:39012)

[19] S. Stević, Asymptotic behaviour of a nonlinear difference equation, Indian J. Pure Appl. Math. 34 (12) (2003), 1681-1687. MR2030114(2005a:39029)

[20] S. STEvić, On the recursive sequence $x_{n+1}=\frac{A}{\prod_{i=0}^{k} x_{n-i}}+\frac{1}{\prod_{j=k+2}^{2(k+1)} x_{n-j}}$, Taiwanese J. Math. 7 (2) (2003), 249-259. MR 1978014 (2004c:39030)

[21] S. Stević, A note on periodic character of a difference equation, J. Differ. Equations Appl. 10 (10) (2004), 929-932. MR2079642 (2005b:39011)

[22] S. Stević, Some open problems and conjectures on difference equations, http://www.mi.sanu.ac.yu, April 29, 2004.

[23] S. Stević, On the recursive sequence $x_{n+1}=\alpha+\frac{x_{n-1}^{p}}{x_{n}^{p}}$, J. Appl. Math \& Computing 18 (1-2) (2005), 229-234. MR2137703 (2005m:39032) 
[24] S. Stević, Global stability and asymptotics of some classes of rational difference equations, J. Math. Anal. Appl. 316 (2006), 60-68. MR2201749(2006i:39026)

[25] S. Stević, On positive solutions of a $(k+1)$-th order difference equation, Appl. Math. Lett. 19 (5) (2006), 427-431. MR2213143

Department of Mathematics, Wake Forest University, Winston-Salem, North CarOLINA 27109

E-mail address: berenhks@wfu.edu

Department of Mathematics, Wake Forest University, Winston-Salem, North CarOLINA 27109

E-mail address: folejd4@wfu.edu

Mathematical Institute of The Serbian Academy of Science, Knez Mihailova 35/I 11000 Beograd, Serbia

E-mail address: sstevic@ptt.yu

E-mail address: sstevo@matf.bg.ac.yu 